\title{
Expression of activator protein-1 (AP-1) family members in breast cancer
}

\author{
Amirhossein Kharman-Biz ${ }^{1,2}$, Hui Gao ${ }^{1}$, Reza Ghiasvand ${ }^{2,3}$, Chunyan Zhao ${ }^{1}$, Kazem Zendehdel ${ }^{2^{*}}$ \\ and Karin Dahlman-Wright ${ }^{1 *}$
}

\begin{abstract}
Background: The activator protein-1 (AP-1) transcription factor is believed to be important in tumorigenesis and altered AP-1 activity was associated with cell transformation. We aimed to assess the potential role of AP-1 family members as novel biomarkers in breast cancer.

Methods: We studied the expression of AP-1 members at the mRNA level in 72 primary breast tumors and 37 adjacent non-tumor tissues and evaluated its correlation with clinicopathological parameters including estrogen receptor (ER), progesterone receptor (PR) and HER2/neu status. Expression levels of Ubiquitin C (UBC) were used for normalization. Protein expression of AP-1 members was assessed using Western blot analysis in a subset of tumors. We used student's t-test, one-way ANOVA, logistic regression and Pearson's correlation coefficient for statistical analyses.

Results: We found significant differences in the expression of AP-1 family members between tumor and adjacent non-tumor tissues for all AP-1 family members except Fos B. Fra-1, Fra-2, Jun-B and Jun-D mRNA levels were significantly higher in tumors compared to adjacent non-tumor tissues $(p<0.001)$, whilst c-Fos and c-Jun mRNA levels were significantly lower in tumors compared with adjacent non-tumor tissues $(p<0.001)$. In addition, Jun-B overexpression had outstanding discrimination ability to differentiate tumor tissues from adjacent non-tumor tissues as determined by ROC curve analysis. Moreover, Fra-1 was significantly overexpressed in the tumors biochemically classified as ERa negative $(p=0.012)$ and PR negative $(p=0.037)$. Interestingly, Fra-1 expression was significantly higher in triple-negative tumors compared with luminal carcinomas $(p=0.01)$.
\end{abstract}

Conclusions: Expression levels of Fra-1 and Jun-B might be possible biomarkers for prognosis of breast cancer.

Keywords: AP-1 family members, Breast cancer, Estrogen receptor, Progesterone receptor

\section{Background}

Breast cancer is the second most common cancer and the most common cancer among women in the world [1]. In addition, breast cancer is a heterogeneous disease that includes several distinct subtypes with distinctive gene expression patterns and different overall survival [2].

The treatment of breast cancer has been greatly advanced in the past decades due to the discovery of specific predictive and prognostic biomarkers that enable the application of more individualized therapies to different molecular subgroups with distinct clinical behavior

\footnotetext{
* Correspondence: kzendeh@tums.ac.ir; karin.dahlman-wright@ki.se

${ }^{2}$ Cancer Research Center, Cancer Institute of Iran, Tehran University of

Medical Sciences, Tehran, Iran

${ }^{1}$ Department of Biosciences and Nutrition, Karolinska Institutet, Stockholm

S-14183, Huddinge, Sweden

Full list of author information is available at the end of the article
}

[3]. Among the established biomarkers for breast cancer, estrogen receptor (ER) is the most powerful predictive marker both in determining prognosis and predicting response to hormone therapies [4]. Recently, human epidermal growth factor receptor 2 (HER2) has also become a routine marker in breast cancer predicting response to HER2 targeted therapy [5-7]. However, there is clearly a need to identify additional biomarkers for breast cancer as all breast cancers do not express ER and/or HER2 and additionally, there is no perfect correlation between these biomarkers and the response to targeted treatment.

It is well established that estrogen signaling and ERs play a central role in the development of breast cancer $[8,9]$. Although many of the known effects of estrogen are mediated via a direct interaction of estrogen with $E R s, E R \alpha$ and ER $\beta$, which regulate the expression of

\section{Biomed Central}


specific sets of genes through a direct interaction with cis-regulatory elements, estrogen-response elements (EREs), of target genes $[9,10]$, it is also well established that ERs interact with DNA indirectly through interaction with other DNA-bound transcription factors such as activator protein-1 (AP-1) complexes [11].

The AP-1 transcription factor is a dimeric complex that includes members of the JUN and FOS protein families. Unlike the JUN family members (c-Jun, Jun-B, Jun-D), the FOS family members (c-Fos, Fra-1, Fra-2 and Fos-B) need to hetrodimerize with members of the JUN family to form transcriptionally active complexes. After dimerization, AP-1 complexes bind to response elements on DNA including TPA response elements (TREs) and cAMP response elements (CREs) in the promoter and enhancer regions of target genes [12,13]. In vitro studies have shown that FOS-JUN heterodimers form more stable complexes and can display stronger DNA-binding activity compared with JUN homodimers $[12,14]$.

Several studies investigated the expression of FOS and JUN family members at the mRNA and protein levels in breast cancer and suggested a role for these proteins as potential biomarkers in breast cancer [14-18]. However, a systemic evaluation of the expression of all AP-1 family members as potential biomarkers in breast cancer is still lacking.

In the present study we focused on the expression of c-Fos, Fra-1, Fra-2, Fos-B, c-Jun, Jun-B and Jun-D in human breast cancer tumors and adjacent non-tumor tissues with the aim to assay the potential of these molecules as novel biomarkers. Their correlation with ER status, progesterone receptor (PR) status, HER2 status, lymph node involvement, stage and grade was further investigated.

\section{Methods}

\section{Tissue collection and tumor specimens}

Tissue samples of 72 primary breast cancer specimens (mean age 48.6 years, median age 46.5 years; range 2485 years) and 37 adjacent non-tumor tissues were available. For 36 cases, paired samples from tumor and adjacent non-tumor tissues were available. Histologically all tumors were classified as invasive ductal and lobular carcinomas. ER, PR and HER2 statuses were available in 70, 62 and 68 cases and were positive in 47, 35 and 14 cases, respectively (Table 1). Receptor status was assessed using Immunohistochemistry (IHC). Fifty-two of the primary breast tumors were lymph node positive and 20 were lymph node negative. Thirty-eight patients were premenopausal and 32 postmenopausal, and for two patients the menopausal status was not available. Forty-two tumors classified as luminal (ER positive and/or PR positive, and HER2 negative), 10 as triple-negative (ER negative, $\mathrm{PR}$ negative and HER2 negative) and 14 as HER2-enriched (HER2 positive) (Table 1). The pathological staging was done as
Table 1 Clinicopathological data

\begin{tabular}{lc}
\hline ER status & \\
Positive (\%) & $47(65.3)$ \\
Negative (\%) & $18(25.0)$ \\
Weak (\%) & $5(6.9)$ \\
Unknown (\%) & $2(2.8)$ \\
PR status & \\
Positive (\%) & $35(48.6)$ \\
Negative (\%) & $25(34.7)$ \\
Weak (\%) & $2(2.8)$ \\
Unknown (\%) & $10(13.9)$ \\
HER2 status & \\
Positive (\%) & $14(19.4)$ \\
Negative (\%) & $54(75.0)$ \\
Weak (\%) & $2(2.8)$ \\
Unknown (\%) & $2(2.8)$ \\
Subtypes of breast cancer & \\
Luminal carcinomas (\%) & $42(63.6)$ \\
Triple-Negative tumors (\%) & $10(15.2)$ \\
HER2-enriched tumors (\%) & $14(21.2)$ \\
\hline
\end{tabular}

recommended by the American Joint Committee on Cancer (AJCC) TNM system. Eight tumors were classified as stage I, 37 as stage II, 25 as stage III and 2 as stage IV. Moreover, 25 patients classified as grade 1, 40 as grade 2, 6 as grade 3 and one as missing. All samples have been provided from the National Tumor Bank of the Cancer Institute of Iran. Informed consent was obtained from all patients who donated samples to the tumor bank. The National Research Ethics Committee of I.R of Iran and the Regional Research Ethics committee of Karolinska Institute approved the study.

\section{Real-time PCR analysis}

RNA was extracted from fresh frozen tissues using RNeasy plus Universal Mini Kits (QIAGEN) according to the manufacturer's instructions. The integrity and concentration of the RNA was assessed using the Agilent Bioanalyzer. Complementary DNA (cDNA) was synthesized using Superscript III First-Strand Synthesis SuperMix (Invitrogen), according to the manufacturer's instructions. One $\mu$ g RNA from each sample was used as starting material for cDNA synthesis.

Real-time PCR was run in triplicate in a $7500 \mathrm{ABI}$ real-time PCR thermocycler (Applied Biosystems). ER $\alpha$ (ESR1), c-Fos and c-Jun mRNA expression were determined by TaqMan assay (Hs00174860_s1), TaqMan assay (Hs04194186_s1) and TaqMan assay (Hs01103582_s1), respectively. The ubiquitin C TaqMan assay (Hs00824723_m1) was used for normalization. The final volume per well for 
TaqMan assays was $15 \mu$ l. SYBR Green assays were used to determine the mRNA expression for Fra-1 (forward primer: GGA GGA AGG AAC TGA and reverse primer: CAC CAA CAT GAA CTC), Fra-2 (forward primer: AAG CTG CAG GCG GAG and reverse primer: CAC CAA CAT GAA CTC), Fos-B (forward primer: GAA CGA AAT AAA CTA and reverse primer: TTT TCT TCC TCC AAC), Jun-B (forward primer: CGC CGA CGG CTT TGT and reverse primer: GGT GTC ACG TGG TTC), Jun-D (forward Primer: CCA GCG AGG AGC AGG and reverse primer: GCT GGT TCT GCT TGT). The final volume per well for SYBR Green assays was $10 \mu \mathrm{l}$. The thermal cycling conditions were $95^{\circ} \mathrm{C}$ for 20 seconds once, then repetitively $95^{\circ} \mathrm{C}$ for 3 seconds and $60^{\circ} \mathrm{C}$ for 30 seconds for all assays.

The expression of 16 candidate endogenous control genes was analyzed by real-time PCR using the TaqMan Endogenous Control Assay on 16 randomly selected samples including 11 tumors and 5 adjacent non-tumor tissues to identify an optimal gene for normalization. The results showed that ubiquitin $\mathrm{C}$ (UBC) displayed the most stable expression among the samples and it was chosen for normalization (data not shown). The mRNA expression was calculated using the $\Delta \mathrm{Ct}$ method by subtracting the average Ct-value of triplicates of selected genes from the average Ct-value of triplicates of the housekeeping gene (UBC) as an internal control.

\section{Western blot analysis}

Frozen tumor tissue was minced and cells were lysed with RIPA/complete mini lysis buffer. Protein extracts were prepared as described previously [14]. Forty $\mu \mathrm{g}$ of protein extract was analyzed by Western blot using c-Fos polyclonal antibody (H-125) sc-7202 (1:200), Fra-1 polyclonal antibody (R-20) sc-605 (1:400), Fra-2 polyclonal antibody (Q-20) sc-604 (1:800), Fos-B polyclonal antibody (102) sc-48 (1:200), c-Jun polyclonal antibody (H-79) sc-1694 (1:400), Jun-B monoclonal antibody (C-11) sc-8051 (1:100) and Jun-D polyclonal antibody (329) sc-74 (1:400). All antibodies were purchased from Santa Cruz biotechnology.

\section{Statistical analysis}

Student's t-test was performed to compare continuous variables between two different categorical clinicopathological characteristics including ER $\alpha, \mathrm{PR}, \mathrm{HER} 2, \mathrm{lymph}$ node status, and menopausal status. In addition, one-way ANOVA was used when comparing a continuous variable with several categorical explanatory variables such as subtypes of breast cancer, staging and BSR grading system. Curve estimation regression and logistic regression models were also fitted with the mRNA expression of AP-1 family members as the outcome variable.

ROC (Receiving Operating Characteristic) curve test was used and the area under the curve (AUC) was calculated to summarize and present the discrimination between tumor and adjacent non-tumor tissues, as the area under the curve defined previously [19]. An arbitrary level of 5\% for statistical significance (two-sided) was considered in all analyses. Statistical analysis was calculated by means of SPSS statistical software version 16 and R software.

\section{Results \\ Differential expression for AP-1 family members in tumors compared to adjacent tissues}

The mRNA expression levels of AP-1 family members in 72 mammary carcinomas and 37 adjacent non-tumor tissues are shown in Figure 1.

Fra-1, Fra-2, Jun-B and Jun-D exhibited significantly higher expression in tumors compared with adjacent tissues $(p<0.001)$. C-Fos and c-Jun mRNA levels were significantly lower in tumors compared with adjacent tissues $(p<0.001)$. Finally, the expression of Fos-B did not differ between tumor tissue and adjacent tissue. Repeating the analysis using a paired design, for the 36 individuals where paired samples were available, produced similar results for the differences in expression levels between tumor tissue and adjacent tissue (Additional file 1: Figure S1).

ROC analysis showed that Jun-B overexpression had outstanding discrimination ability to differentiate tumor tissue from adjacent non-tumor tissue $(A U C=0.983)$ (Figure 2). In addition, Jun-D, Fra-2 and Fra-1 had acceptable discrimination abilities (AUC $=0.894,0.811$ and 0.782, respectively) (Table 2 ).

Pearson's correlation analysis was performed pairwise for all seven AP-1 family members the expressions of which were analyzed in this cross sectional study (Figure 3). In this analysis, c-Jun and c-Fos showed strong positive correlation $(r=0.78)$. In addition, there were moderate positive correlations between Jun-B and Jun-D ( $r=0.63)$, and between Fra-2 and Jun-D $(r=0.65)$. Furthermore, Jun-D and Jun-B displayed moderate positive correlation to $E R \alpha$ mNRA levels in ER $\alpha$ positive tumors $(n=47)$ (Additional file 1: Figure S2A and S2B).

\section{Association of mRNA expression levels with protein expression levels}

Western blot analysis on six tumor samples demonstrated correlation between mRNA and protein levels for Fra-1, Fra-2, Jun-B and Jun-D (Figure 4). Figure 4A and D show the Western blot analysis for Fra-1, Fra-2, Jun-B and Jun-D, respectively. Figure $4 \mathrm{~B}, \mathrm{C}, \mathrm{E}$ and $\mathrm{F}$ show correlations between mRNA and protein expression levels for Fra-1, Fra-2, Jun-B and Jun-D, respectively. The samples for these analyses were chosen based on that they displayed differential expression of AP-1 family members to allow correlation between mRNA expression levels and protein levels. The protein level of c-Jun did not perfectly correlate with mRNA expression level (data not shown). For c-Fos 

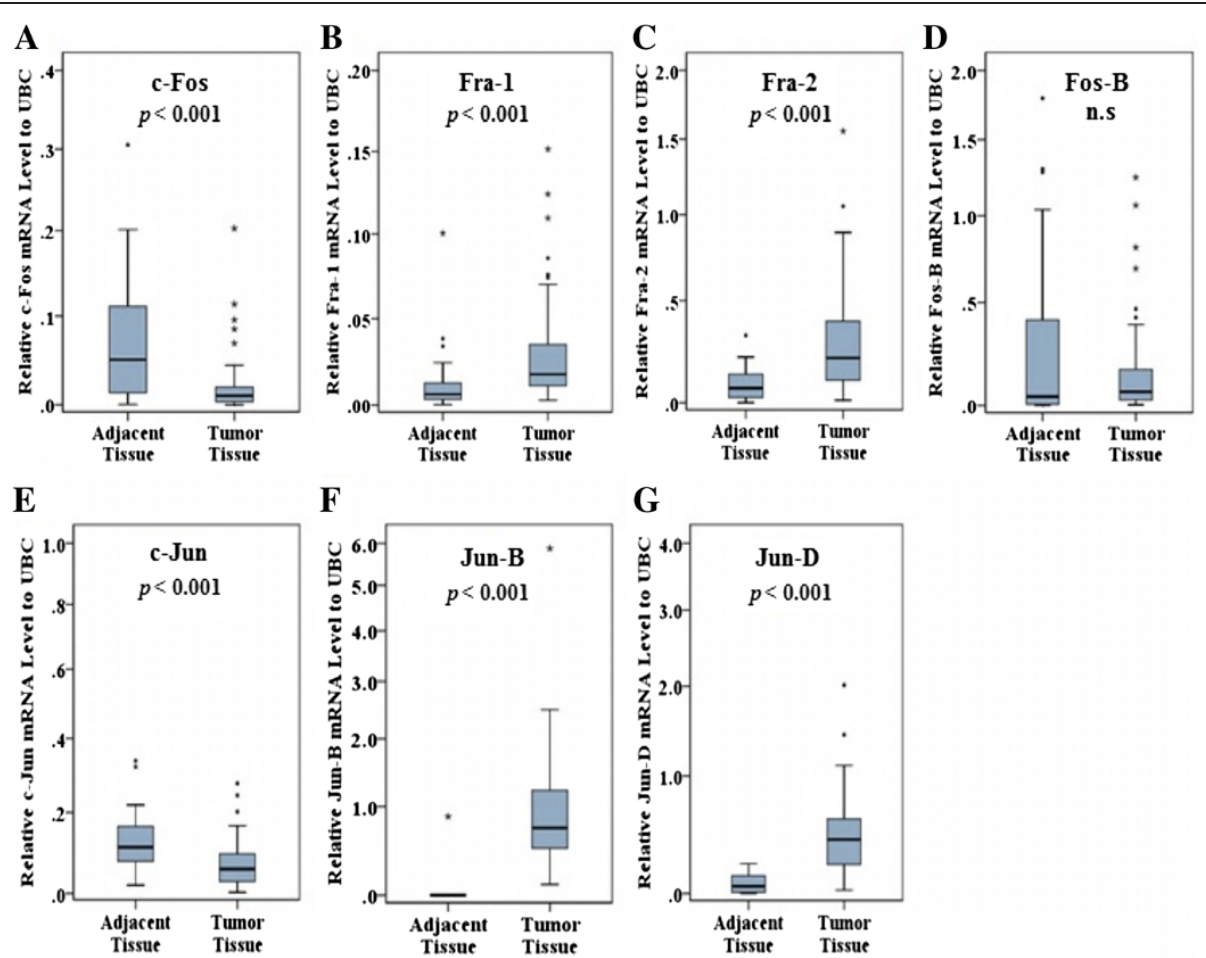

Figure 1 Expression of AP-1 family members comparing tumor and adjacent tissues. The expression of Fra-1 (B), Fra-2 (C), Jun-B (F) and Jun-D (G) are significantly higher in tumors compared with adjacent tissues $(p<0.001)$, whereas the expression of $c$-Fos $(\mathbf{A})$ and $c-J u n(\mathbf{E})$ are significantly lower in tumors compared with adjacent tissues $(p<0.001)$. A non-paired model was applied. Gene expression (y-axis) was quantified by real-time PCR and normalized to UBC.

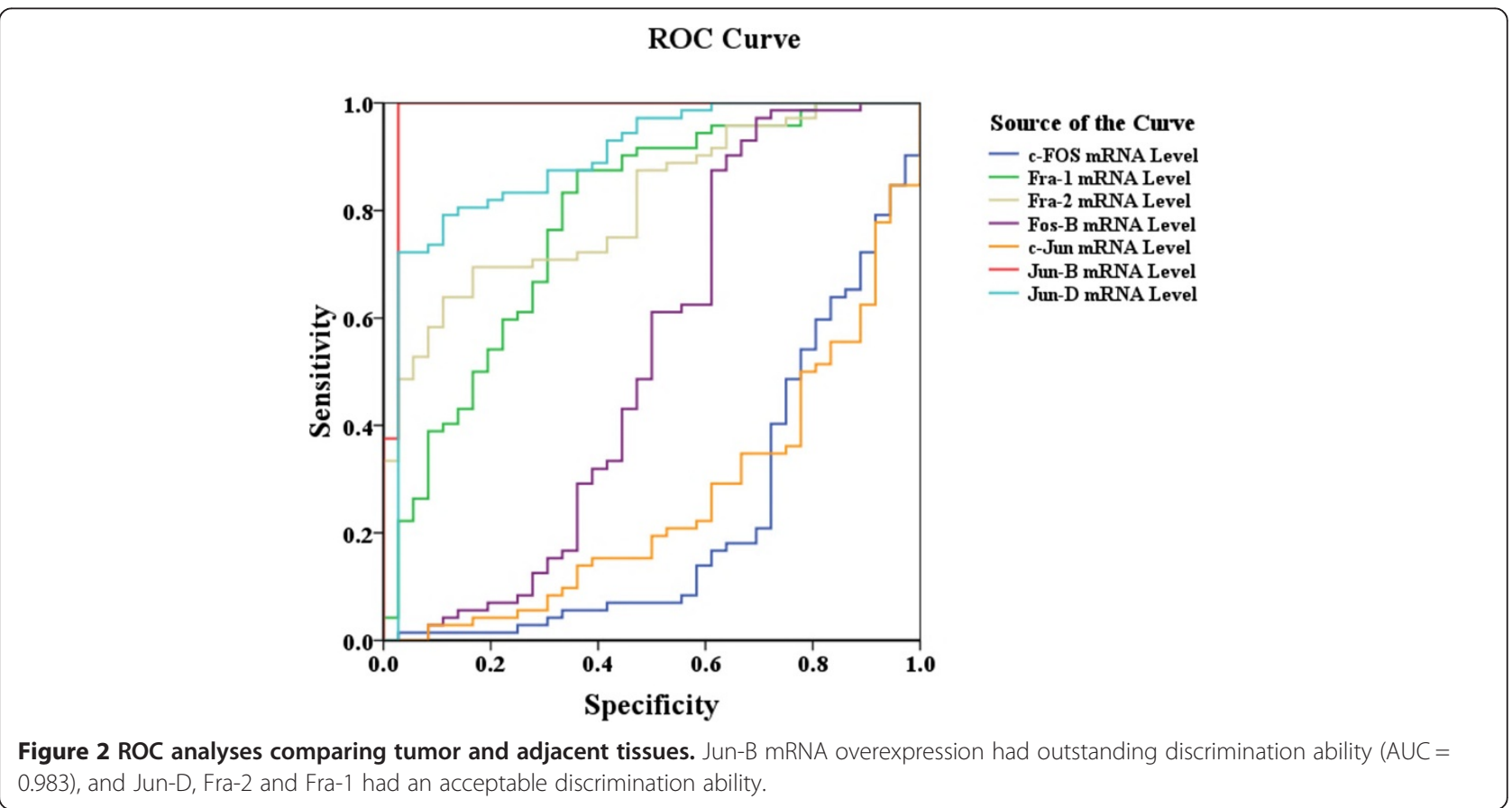


Table 2 Area Under the Curve (AUC) of ROC analysis

\begin{tabular}{cc}
\hline Test result variable(s) & AUC \\
\hline Jun-B mRNA Level & 0.983 \\
Jun-D mRNA Level & 0.894 \\
Fra-2 mRNA Level & 0.811 \\
Fra-1 mRNA Level & 0.782 \\
Fos-B mRNA Level & 0.522 \\
c-Jun mRNA Level & 0.258 \\
c-Fos mRNA Level & 0.230 \\
\hline
\end{tabular}

The minimum value of $A \cup C$ is 0.5 ; the maximum is 1 . Jun-B overexpression has outstanding discrimination ability, and also Jun-D, Fra-2 and Fra-1 have acceptable discrimination abilities.

and Fos-B, protein expression was undetectable presumably due to low expression as we could detect positive controls for these proteins in the assays (data not shown).

Fra-1 is overexpressed in triple-negative breast tumors We classified the breast cancer tumors into different subtypes based on IHC staining as defined by O'Brien et al. [20]. ANOVA analysis revealed a significant difference among subtypes of breast cancer for Fra-1 expression $(p=0.039)$ (Table 3 and Figure 5C). Further analysis showed that Fra-1 mRNA expression was significantly higher in triple-negative tumors compared with luminal carcinomas $(p=0.01)$. However, the difference between the transcript levels in TNBC and HER2-enriched breast cancers was not statistically significant. When comparing the mRNA expression of AP-1 family members with known molecular markers of breast cancer, Fra-1 was significantly higher expressed in tumors classified as $\mathrm{ER} \alpha$ negative tumors compared with $\mathrm{ER} \alpha$ positive tumors $(p=0.012)$ (Figure 5A). Similarly, the expression level of Fra-1 was significantly higher in PR negative tumors compared with PR positive tumors $(p=0.037)$ (Figure 5B). Additionally, there was no correlation between the mRNA expression of other JUN and FOS family members and hormone receptor status.

\section{Association of AP-1 mRNA levels with clinical characteristics}

We investigated the mRNA expression of AP-1 family members in relation to clinical characteristics such as clinical staging, histological grading, lymph node and menopausal statuses and age. We found significant associations between the expression of c-Fos and Fos-B with histological grading, with about 40 percent lower expression of c-Fos [Odds ratio $(\mathrm{OR})=0.62,95 \%$ confidence interval (CI): $0.42-0.94]$ and Fos-B $(\mathrm{OR}=0.62$, 95\% CI: 0.41 - 0.93) in grade two compared with grade one (Table 4). In addition, there were significant associations between the expressions of c-Fos $(p=0.02)$, Fra-1 $(p=0.05)$ and Jun-B $(p<0.0 .1)$ with stage, where the lowest expression levels were observed for the combined stages III and IV compared with the other stages. Moreover,

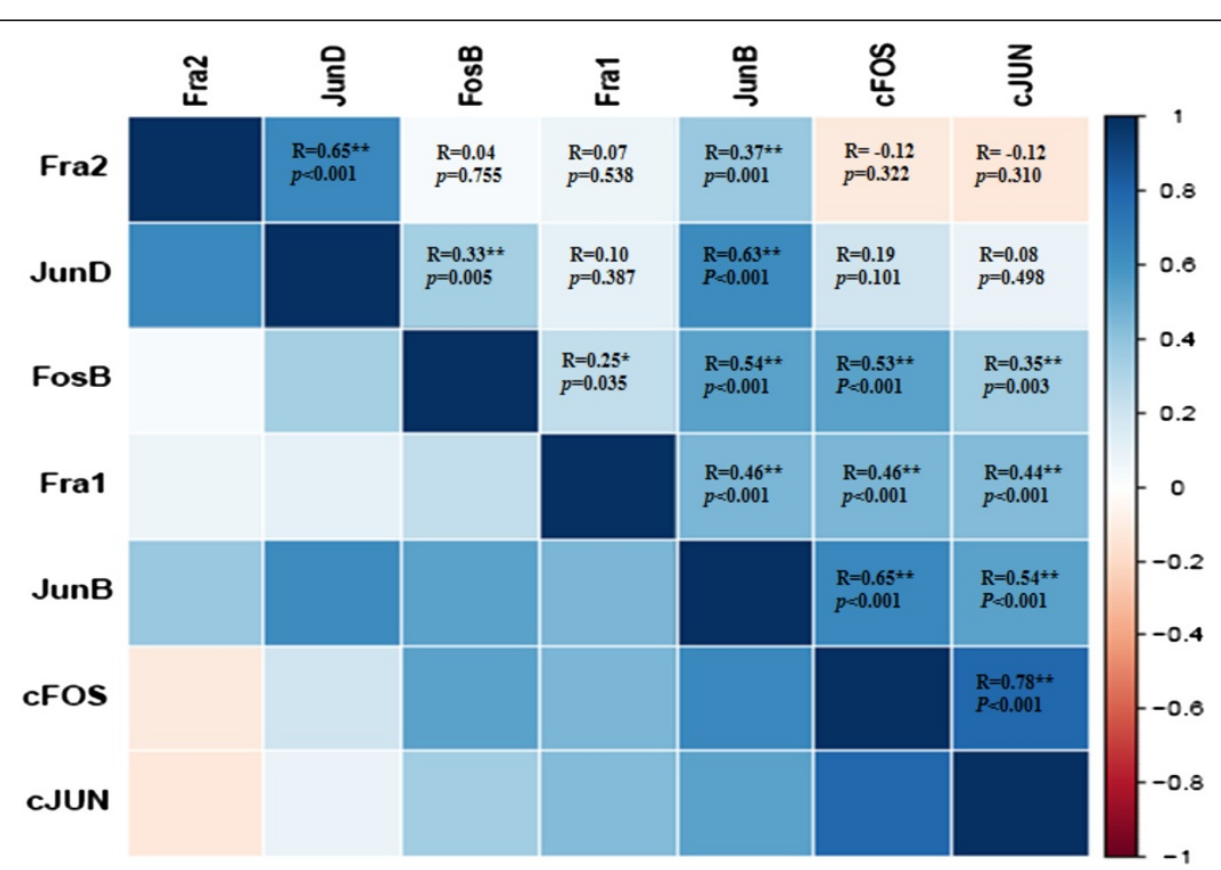

Figure 3 Heat map of the Pearson's correlation matrix for the mRNA expression of AP-1 members. Pairwise correlation analyses were performed for all assayed mRNAs. Blue represents positive correlation for a given gene pair, and red represents negative correlation. C-Jun and c-Fos showed strong positive correlation $(r=0.78)$. In addition, there were moderate positive correlations between Jun-B and Jun-D ( $r=0.63)$, and between Fra-2 and Jun-D ( $r=0.65)$. 


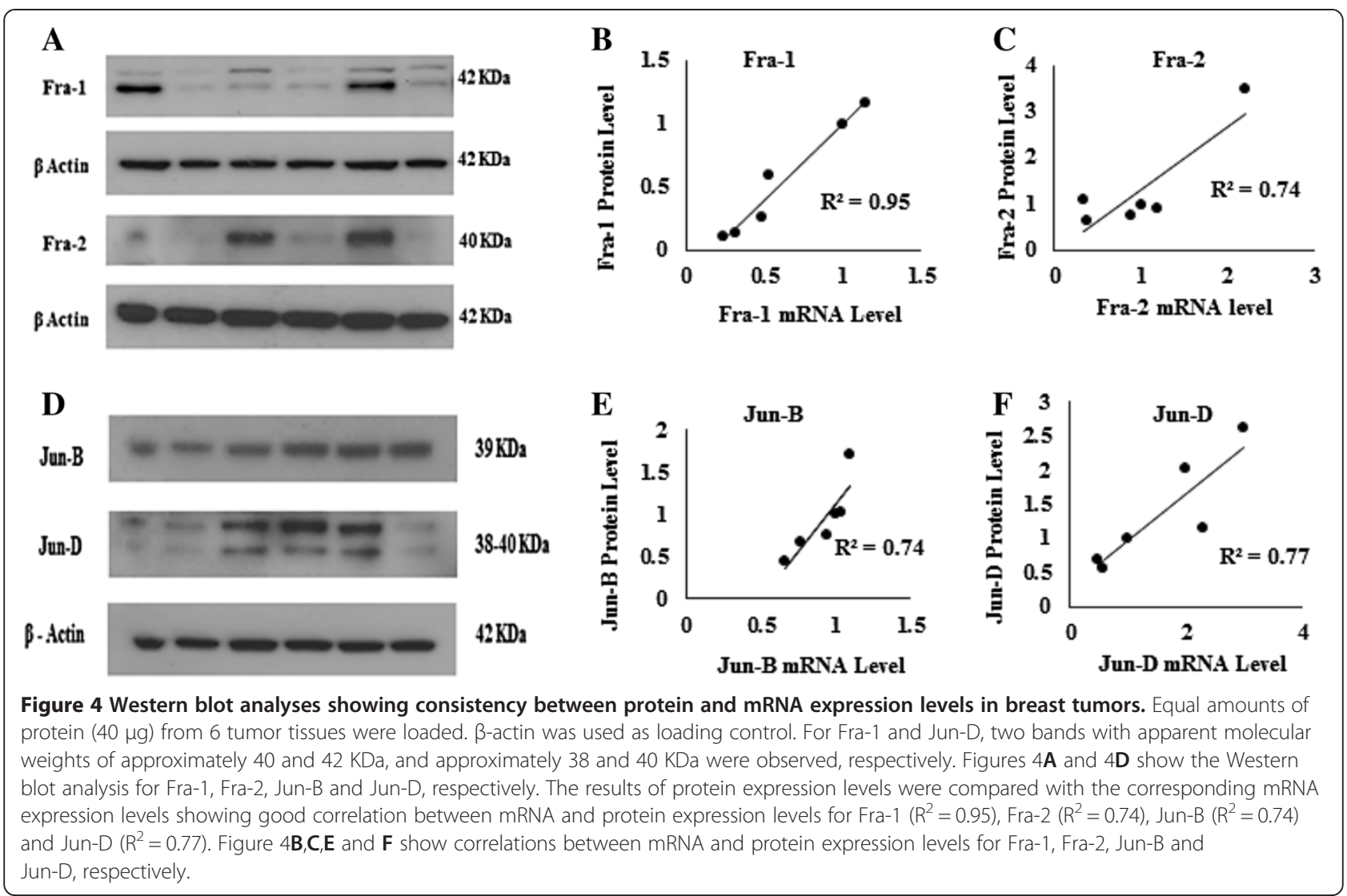

when comparing the mRNA expression of AP-1 family members according to menopausal status, we found a higher expression of Jun-D in premenopausal patients compared with postmenopausal patients $(\mathrm{OR}=0.55$, 95\% CI: 0.30 - 0.99) (Table 5). Furthermore, we observed more than $50 \%$ lower expression of Fra-1 and Jun-B in tumors with lymph node involvement compared to tumors without lymph node involvement, where the ORs were 0.43 (95\% CI: $0.22-0.83$ ) and 0.43 (95\% CI: 0.20-0.92), respectively (Tables 4 and 5). Additionally, since age might play a role as a potential confounder in the association of AP-1 mRNA levels with clinical characteristics, we correlated the mRNA expression levels of selected genes with age, and we found no correlation between mRNA expression levels of selected genes and age (data not shown).

\section{Discussion}

In the present study, we conducted a comprehensive analysis of mRNA expression levels of AP-1 family

Table 3 Analyses of mRNA expression of AP-1 family members in relation to subtypes of breast cancer

\begin{tabular}{lcccc}
\hline & \multicolumn{3}{c}{ Mean $( \pm$ SD) } & $\begin{array}{c}\text { P Value } \\
\text { Variable }\end{array}$ \\
\cline { 2 - 4 } c-Fos mRNA Level & Luminal tumors & Triple-negative tumors & HER2-enriched tumors & (Among subtypes) \\
Fra-1 mRNA Level & $0.024 \pm 0.038$ & $0.012 \pm 0.014$ & $0.011 \pm 0.009$ & 0.834 \\
Fra-2 mRNA Level & $0.330 \pm 0.439$ & $0.051 \pm 0.043$ & $0.026 \pm 0.019$ & $0.039^{*}$ \\
Fos-B mRNA Level & $0.189 \pm 0.283$ & $0.381 \pm 0.253$ & $0.197 \pm 0.155$ & 0.238 \\
c-Jun mRNA Level & $0.076 \pm 0.065$ & $0.090 \pm 0.120$ & $0.082 \pm 0.108$ & 0.214 \\
Jun-B mRNA Level & $0.885 \pm 0.612$ & $0.044 \pm 0.027$ & $0.068 \pm 0.037$ & 0.460 \\
Jun-D mRNA Level & $0.475 \pm 0.407$ & $1.327 \pm 1.655$ & $0.983 \pm 0.695$ & 0.708 \\
\hline
\end{tabular}

One-way ANOVA was used to calculate the $\mathrm{p}$ values among different subtypes. Fra-1 expression showed a significant difference among subtypes.

*Post-hoc analysis using LSD revealed that Fra-1 expression was significantly higher in triple-negative tumors compared with luminal carcinomas ( $p=0.01$ ). In addition, Fra-1 expression was marginally significantly higher in triple-negative tumors compared with HER2-enriched $(p=0.067)$. 


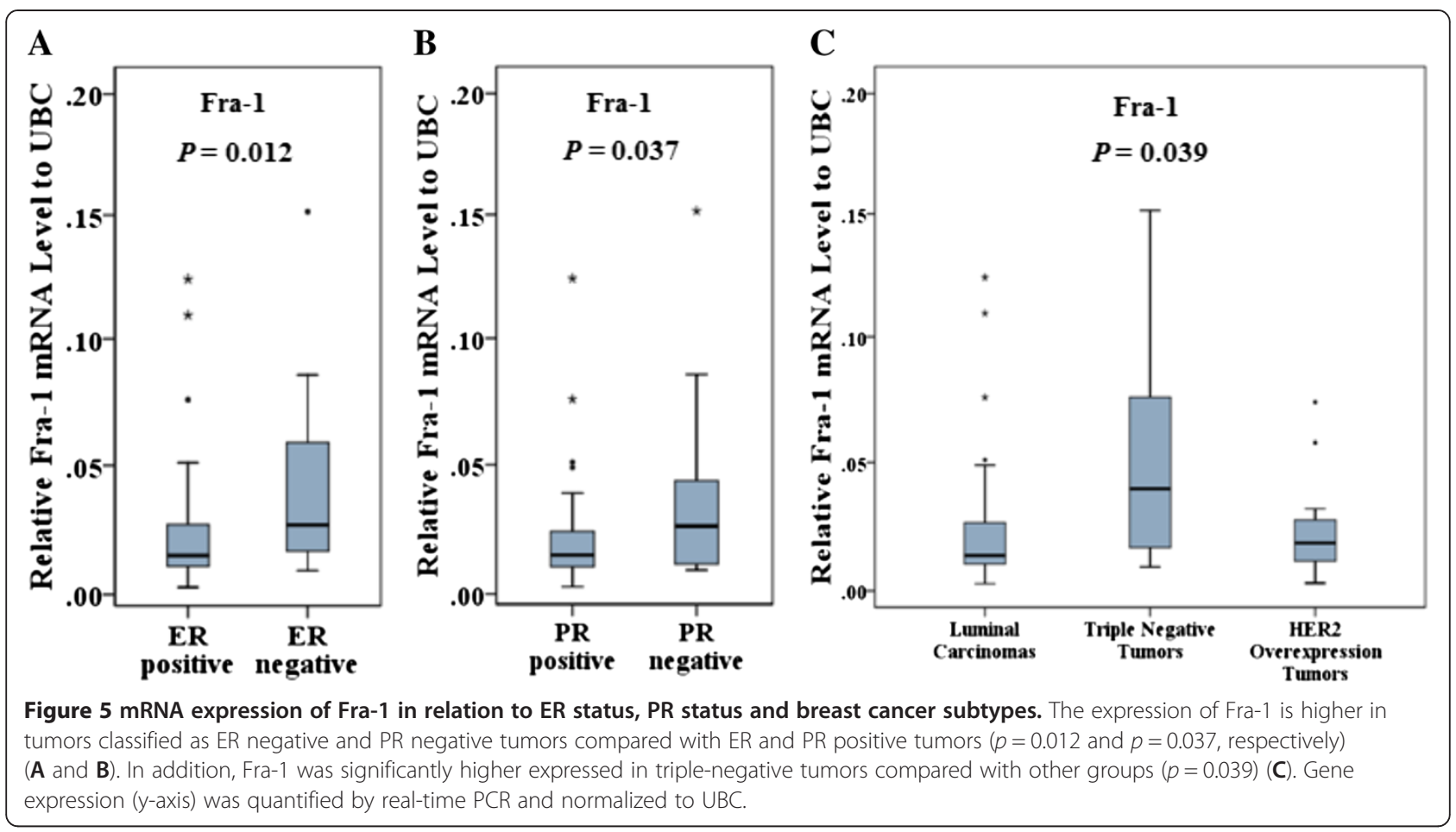

Table 4 ORs and 95\% Cls for FOS member mRNA expression in tumors with clinicopathological parameters

\begin{tabular}{|c|c|c|c|c|}
\hline \multirow[t]{2}{*}{ Parameter } & \multicolumn{4}{|c|}{ OR $(95 \% \mathrm{Cl})$} \\
\hline & c-Fos & Fra-1 & Fra-2 & Fos-B \\
\hline \multicolumn{5}{|c|}{ Menopausal status } \\
\hline Pre-menopause & 1 & 1 & 1 & 1 \\
\hline Post-menopause & $0.95(0.71-1.26)$ & $1.24(0.72-2.14)$ & $0.78(0.48-1.26)$ & $0.84(0.60-1.19)$ \\
\hline$P$ value & 0.71 & 0.43 & 0.32 & 0.33 \\
\hline \multicolumn{5}{|c|}{ Lymph node status } \\
\hline Negative & 1 & 1 & 1 & 1 \\
\hline Positive & $0.72(0.50-1.06)$ & $0.43(0.22-0.83)$ & $0.83(0.49-1.41)$ & $1.00(0.70-1.46)$ \\
\hline$P$ value & 0.09 & $0.01^{*}$ & 0.49 & 0.98 \\
\hline \multicolumn{5}{|l|}{ Stage } \\
\hline । & 1 & 1 & 1 & 1 \\
\hline$\|$ & $1.18(0.70-2.01)$ & $0.71(0.29-1.75)$ & $0.83(0.38-1.82)$ & $1.02(0.58-1.78)$ \\
\hline III and IV & $0.75(0.44-1.26)$ & $0.37(0.14-0.99)$ & $0.78(0.35-1.76)$ & $0.86(0.48-1.53)$ \\
\hline$P$ value & $0.02^{*}$ & $0.05^{*}$ & 0.84 & 0.64 \\
\hline \multicolumn{5}{|l|}{ Grade } \\
\hline 1 & 1 & 1 & 1 & 1 \\
\hline 2 & $0.62(0.42-0.94)$ & $0.99(0.56-1.76)$ & $1.14(0.62-1.90)$ & $0.62(0.41-0.93)$ \\
\hline 3 & $0.70(0.37-1.29)$ & $1.15(0.42-3.17)$ & $1.23(0.50-3.04)$ & $0.73(0.37-1.44)$ \\
\hline$P$ value & $0.04^{*}$ & 0.95 & 0.83 & $0.05^{*}$ \\
\hline
\end{tabular}

ORs and Cls from logistic regression model, stratified on some parameters including menopausal and lymph node statuses, grade and stage. Abbreviations: $\mathrm{OR}$, Odds ratio; $\mathrm{Cl}$, Confidence interval.

"Statistically significant $(p<0.05$ in two-tailed test). 
Table 5 ORs and $95 \%$ Cls for JUN member mRNA expression with clinicopathological parameters

\begin{tabular}{cccc}
\hline Parameter & \multicolumn{3}{c}{ OR $(95 \% \mathrm{Cl})$} \\
\cline { 2 - 4 } & C-Jun & Jun-B & Jun-D \\
\hline
\end{tabular}

\section{Menopausal status}

Pre-menopause

1

Post-menopause

$P$ value

$0.80(0.49-1.3)$

0.36

Lymph node status

Negative

Positive

$P$ value

$$
0.84(0.48-1.45)
$$$$
0.52
$$

Stage

\section{I}

II

III and IV

$P$ value

Grade

1

2

3

$P$ value

\section{1 \\ 1.49 (0.67-3.30) \\ 0.881 (0.40-1.92) \\ 0.14}

1

$0.66(0.37-1.192)$

$0.54(0.21-1.36)$

0.26
$0.43(0.20-0.92)$

$0.03^{*}$

$0.95(0.52-1.71)$

1

$0.77(0.42-1.41)$

0.40

$0.55(0.30-0.99)$

$0.04^{*}$

0.86

1

$0.65(0.22-1.97) \quad 1.40(0.59-3.33)$

$0.25(0.07-0.82) \quad 0.93(0.39-2.23)$

0.37

$$
<0.01^{* *}
$$

$0.56(0.28-1.13) \quad 1.11(0.63-1.95)$ $0.65(0.20-2.13) \quad 0.98(0.37-2.63)$

ORs and Cls from logistic regression model, stratified on parameters including menopausal and lymph node statuses, grade and stage.

Abbreviations: $\mathrm{OR}$, Odds ratio; $\mathrm{Cl}$, Confidence interval.

*Statistically significant ( $p<0.05$ in two-tailed test).

${ }^{* *}$ Statistically significant ( $p<0.01$ in two-tailed test).

members in 72 primary breast tumors and 37 adjacent tissues. However, this approach has its limitations when addressing the correlation of biomarkers with tumor development. Future studies could be designed to also allow sampling of normal breast tissue. However, also this approach has limitations because the percentage of epithelial cells is generally higher in breast cancer tissue. Analysis of samples after microdissection of the corresponding cell types from tumor and adjacent tissue as well as normal breast tissue would constitute an important development to identify tumor biomarkers.

The median age of the studied population at the time of diagnosis was 46.5 years. This is lower than the median age for breast cancer diagnosis worldwide [21]. The lower median age at diagnosis is consistent with the established younger age at diagnosis of breast cancer in developing countries [22,23], which is believed to relate to the younger population in developing countries [24], which cause higher number of young patients.

In this study we observed that c-Fos and c-Jun were significantly lower expressed in tumors compared with adjacent tissues. Whereas, Fra-1, Fra-2, Jun-B and Jun-D were significantly higher expressed in tumors compared with adjacent tissue. Finally, our analysis suggests that two members of the AP- 1 family members, Fra- 1 and Jun-B are associated with clinical parameters and thus might provide novel markers for breast cancer. Taking into consideration the efficiency of the different PCR reactions, we estimate that across all samples, Jun $B$ is expressed at the highest level followed by Jun-D. C-Jun and c-Fos and Fra- 2 are expressed at about equal levels while Fos-B and Fra-1 are expressed at a lower level as determined from this analysis (Data not shown).

Consistent with previous reports, our results revealed that c-Fos and c-Jun are expressed at a higher level in adjacent tissues compared with tumors [13,25]. Smith et al investigated the expression and activity of the AP-1 complex in human mammary epithelial cells (HMECs) at different stages including normal, immortal, oncogenetransformed and cancer. They showed that normal cells and immortal HMECs have higher mRNA and protein expression levels of c-Fos and c-Jun compared with human breast cancers [25].

Our results showing high expression of Fra-1 in breast tumors and its differential expression between ER $\alpha$ positive and ER $\alpha$ negative tumors are consistent with previous studies $[14,17,26,27]$. Fra-1 was significantly higher expressed in triple-negative tumors compared with other groups (Figure 5C). However, the difference between the transcript levels in TNBC and HER2-enriched breast cancers was only marginally statistically significant $(p=0.067)$. Thus, Fra-1 would not appear to define an auxiliary diagnostic marker for TNBC, which is clearly need in light of the remarkable challenges that this breast cancer subtype presents to researchers and clinicians. In vitro studies have shown that Fra-1 expression is associated with cell motility, proliferation and invasiveness [28]. However, our findings showed that the expression of Fra-1 was significantly higher in nodal negative tumors compared with nodal positive tumors, and furthermore no correlation was observed between Fra-1 expression and metastasis status. In a cohort study Schroder et al. investigated Fra-2 mRNA expression in 167 patients and found significant correlations of high Fra-2 expression with younger age, nodal involvement, high grading and ER negative tumors [18]. However, our findings did not show any association of Fra-2 mRNA expression with ER or nodal status and grading.

Our results demonstrated that there was a significant association between increased Jun-B mRNA expression and reduced tumor size and tumor stage. Moreover, Jun-B mRNA expression was significantly higher in nodal negative tumors compared with nodal positive tumors. These findings imply that increased Jun-B expression could be related to a less aggressive tumor behavior. These results are similar with previous studies suggesting that Jun-B could have a role as a tumor suppressor [15]. 
Milde-Langosch et al. investigated Fos-B mRNA and protein expression in human breast tumors and normal breast tissues and showed that Fos-B was highly expressed in normal lobules and ducts with carcinomas frequently displaying loss of expression and weak immunostaining. They showed that Fos-B might be necessary for normal proliferation and differentiation of mammary epithelial cells and also Fos-B is down-regulated in poorly differentiated breast tumors [29]. Our results, displaying a marginally significant association between increased Fos-B mRNA expression and well-differentiated status are consistent with this report. In contrast, our findings did not show a significant difference in Fos-B expression between tumors and adjacent tissues.

There was no clear correlation between expression of AP-1 members in tumors versus surrounding tissues and the expression in higher grade and higher stage tumors. For example c-Fos was lower expressed in tumors versus surrounding tissues and the expression was also lower in higher grade and higher stage tumors while Jun-B expression was higher in tumors compared to surrounding tissues while the expression was lower in higher grade and stage tumors.

In this study we focus on the JUN and FOS family members of the AP-1 family of transcription factors. However additionally, members of the ATF (activating transcription factor) and MAF (musculoaponeurotic fibrosarcoma) protein families have been shown to contribute to this complex and could thus be considered as potential additional biomarkers in breast cancer.

\section{Conclusions}

Overall, our findings reveal, among others, that Fra-1 mRNA levels are higher in ER $\alpha$ negative and PR negative breast cancer tumors. In addition, Jun-B overexpression has outstanding discrimination ability $(\mathrm{AUC}=0.983$ ) to differentiate tumor tissues from adjacent tissues. We therefore suggest that AP-1 family members should be further evaluated in larger cohorts as possible biomarkers in breast cancer.

\section{Additional file}

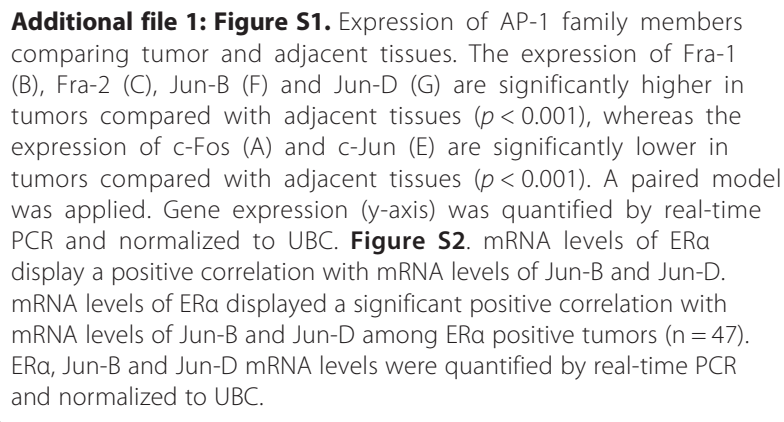

\section{Abbreviations}

AP-1: Activator protein-1; ER: Estrogen receptor; PR: Progesterone receptor; HER2: Human epithermal growth factor receptor 2; UBC: Ubiquitin C; mRNA: Messenger ribonucleic acid; cDNA: Complementary deoxyribonucleic acid; Fra-1: Fos-related antigen 1; Fra-2: Fos-related antigen 2; RTqPCR: Reverse transcriptase quantitative polymerase chain reaction; SBR grading system: Scarff, Bloom, Richardson, grading system; ROC: Receiving operating characteristic; AUC: Area under the curve; OR: Odds ratio; Cl: Confidence interval; HMECs: Human mammary epithelial cells; ATF: Activating transcription factor; MAF: Musculoaponeurotic fibrosarcoma.

\section{Competing interests}

The authors declare that they have no competing interest.

\section{Authors' contributions}

AKH, HG, CZ, KZ and KDW designed the study. AKH performed RNA extraction, GRT-PCR and Western blot analysis with supervision of HG and $\mathrm{KDW}$. AKH, HG, RGH and KZ conducted the statistical analysis. AKH drafted the manuscript and all authors revised and approved it. KDW supervised the overall study. All authors read and approved the final manuscript.

\section{Acknowledgements}

We wish to thank members of Professor Karin Dahlman-Wright group for sharing materials and for fruitful discussions. In addition, we wish to thank Bioinformatics and Expression Analysis core facility (BEA) for performing the Agilent Assay. This study was supported by the Center for Biosciences, Karolinska Institutet, and Cancer Research Center of Cancer Institute of Iran, Tehran university of Medical Sciences. Biological Material was provided by the IRAN NATIONAL TUMOR BANK, which is funded by Cancer Institute of Tehran University, for Cancer Research.

\section{Author details}

'Department of Biosciences and Nutrition, Karolinska Institutet, Stockholm S-14183, Huddinge, Sweden. ${ }^{2}$ Cancer Research Center, Cancer Institute of Iran, Tehran University of Medical Sciences, Tehran, Iran. ${ }^{3}$ Department of Biostatistics, Institute of Basic Medical Sciences, University of Oslo, Oslo, Norway.

Received: 5 April 2013 Accepted: 25 September 2013

Published: 28 September 2013

\section{References}

1. Parkin DM, Bray F, Ferlay J, Pisani P: Global cancer statistics, 2002. CA Canc J Clin 2005, 55:74-108.

2. Sorlie T, Perou CM, Tibshirani R, Aas T, Geisler S, Johnsen H, Hastie T, Eisen $M B$, van de Rijn M, Jeffrey SS, et al: Gene expression patterns of breast carcinomas distinguish tumor subclasses with clinical implications. Proc Natl Acad Sci U S A 2001, 98(19):10869-10874.

3. Sawyers CL: The cancer biomarker problem. Nature 2008, 452(7187):548-552.

4. Potter JD, Cerhan JR, Sellers TA, MCGovern PG, Drinkard C, Kushi LR, Folsom AR: Progesterone and estrogen receptors and mammary neoplasia in the lowa women's health study: how many kinds of breast cancer are there? Canc Epidemiol Biomarkers Prev 1995, 4(4):319-326.

5. Hanna W: Testing for HER2 status. Oncology 2001, 61(Suppl 2):22-30.

6. Weigel MT, Dowsett M: Current and emerging biomarkers in breast cancer: prognosis and prediction. Endocr Relat Canc 2010, 17(4):R245-R262.

7. Ali S, Coombes RC: Endocrine-responsive breast cancer and strategies for combating resistance. Nat Rev Canc 2002, 2(2):101-112.

8. Pike MC, Spicer DV, Dahmoush L, Press MF: Estrogens, progestogens, normal breast cell proliferation, and breast cancer risk. Epidemiol Rev 1993, 15(1):17-35.

9. Huang W-Y, Newman B, Millikan RC, Schell MJ, Hulka BS, Moorman PG: Hormone-related Factors and Risk of Breast Cancer in Relation to Estrogen Receptor and Progesterone Receptor Status. Am J Epidemiol 2000, 151(7):703-714.

10. Dahlman-Wright K, Cavailles V, Fuqua SA, Jordan VC, Katzenellenbogen JA, Korach KS, Maggi A, Muramatsu M, Parker MG, Gustafsson JA: International Union of Pharmacology. LXIV. Estrogen receptors. Pharmacol Rev 2006, 58(4):773-781 
11. Kushner PJ, Agard DA, Greene GL, Scanlan TS, Shiau AK, Uht RM, Webb P: Estrogen receptor pathways to AP-1. J Steroid Biochem Mol Biol 2000 74(5):311-317.

12. Milde-Langosch $\mathrm{K}$, Roder $\mathrm{H}$, Andritzky B, Aslan B, Hemminger G, Brinkmann A, Bamberger CM, Loning T, Bamberger AM: The role of the AP-1 transcription factors C-Fos, FosB, Fra-1 and Fra- 2 in the invasion process of mammary carcinomas. Breast Canc Res Treat 2004, 86(2):139-152.

13. Angel P, Karin M: The role of Jun, Fos and the AP-1 complex in cell-proliferation and transformation. Biochim Biophys Acta 1991, 1072(2-3):129-157.

14. Bamberger AM, Methner C, Lisboa BW, Stadtler C, Schulte HM, Loning T, Milde-Langosch K: Expression pattern of the AP-1 family in breast cancer: association of fosB expression with a well-differentiated, receptorpositive tumor phenotype. Int J Canc 1999, 84(5):533-538.

15. Langer $S$, Singer CF, Hudelist G, Dampier B, Kaserer K, Vinatzer U, Pehamberger $\mathrm{H}$, Zielinski C, Kubista E, Schreibner M: Jun and Fos family protein expression in human breast cancer: correlation of protein expression and clinicopathological parameters. Eur J Gynaecol Oncol 2006, 27(4):345-352.

16. Milde-Langosch K, Janke S, Wagner I, Schroder C, Streichert T, Bamberger AM, Janicke F, Loning T: Role of Fra-2 in breast cancer: influence on tumor cell invasion and motility. Breast Canc Res Treat 2008, 107(3):337-347.

17. Logullo AF, Stiepcich MM, Osorio CA, Nonogaki S, Pasini FS, Rocha RM, Soares FA, Brentani MM: Role of Fos-related antigen 1 in the progression and prognosis of ductal breast carcinoma. Histopathology 2011, 58(4):617-625.

18. Schroder C, Schumacher U, Muller V, Wirtz RM, Streichert T, Richter U, Wicklein $\mathrm{D}$, Milde-Langosch K: The transcription factor Fra-2 promotes mammary tumour progression by changing the adhesive properties of breast cancer cells. Eur J Canc 2010, 46(9):1650-1660.

19. LaValley MP: Logistic regression. Circulation 2008, 117(18):2395-2399.

20. O'Brien KM, Cole SR, Tse CK, Perou CM, Carey LA, Foulkes WD, Dressler LG, Geradts J, Millikan RC: Intrinsic breast tumor subtypes, race, and long-term survival in the carolina breast cancer study. Clin Canc Res 2010, 16(24):6100-6110.

21. El Saghir NS, Seoud M, Khalil MK, Charafeddine M, Salem ZK, Geara FB, Shamseddine Al: Effects of young age at presentation on survival in breast cancer. BMC Canc 2006, 6:194.

22. Montazeri A, Ebrahimi M, Mehrdad N, Ansari M, Sajadian A: Delayed presentation in breast cancer: a study in Iranian women. BMC Women's Health 2003, 3(1):4.

23. Mousavi M, Montazeri A, Mohagheghi MA, Mousavi Jarrahi A, Harirchi I, Najafi M, Ebrahimi M: Breast Cancer in Iran: An Epidemiological Review. Breast J 2007, 13(4):383-391.

24. Mousavi SM, Zheng T, Dastgiri S, Miller AB: Age distribution of breast cancer in the middle East, implications for screening. Breast J 2009, 15(6):677-679.

25. Smith LM, Birrer MJ, Stampfer MR, Brown PH: Breast cancer cells have lower activating protein 1 transcription factor activity than normal mammary epithelial cells. Canc Res 1997, 57(14):3046-3054.

26. Chiappetta G, Ferraro A, Botti G, Monaco M, Pasquinelli R, Vuttariello E, Arnaldi L, Di Bonito M, D'Aiuto G, Pierantoni GM, et al: FRA-1 protein overexpression is a feature of hyperplastic and neoplastic breast disorders. BMC Canc 2007, 7:17.

27. Belguise K, Milord S, Galtier F, Moquet-Torcy G, Piechaczyk M, Chalbos D: The PKCtheta pathway participates in the aberrant accumulation of Fra-1 protein in invasive ER-negative breast cancer cells. Oncogene 2012, 31(47):4889-4897.

28. Belquise K, Kersual N, Galtier F, Chalbos D: FRA-1 expression level regulates proliferation and invasiveness of breast cancer cells. Oncogene 2005, 24(8): 1434-1444

29. Milde-Langosch K, Kappes H, Riethdorf S, Loning T, Bamberger AM: FosB is highly expressed in normal mammary epithelia, but down-regulated in poorly differentiated breast carcinomas. Breast Canc Res Treat 2003, 77(3):265-275

doi:10.1186/1471-2407-13-441

Cite this article as: Kharman-Biz et al.: Expression of activator protein-1 (AP-1) family members in breast cancer. BMC Cancer 2013 13:441.

\section{Submit your next manuscript to BioMed Central and take full advantage of:}

- Convenient online submission

- Thorough peer review

- No space constraints or color figure charges

- Immediate publication on acceptance

- Inclusion in PubMed, CAS, Scopus and Google Scholar

- Research which is freely available for redistribution 